\title{
A Novel Role of SIRT1/ FGF-21 in Taurine Protection Against Cafeteria Diet-Induced Steatohepatitis in Rats
}

\author{
Azza H. Abd Elwahaba Basma K. Ramadan ${ }^{a}$ Mona F. Schaalan ${ }^{b}$ \\ Amina M. Tolbac \\ aDepartment of Physiology, Faculty of Medicine for Girls (Cairo), Al-Azhar University, Cairo, \\ ${ }^{b}$ Department of Biochemistry Faculty of Pharmacy, Misr International University, Cairo, 'Department of \\ Anatomy, Faculty of Medicine for Girls (Cairo), Al-Azhar University, Cairo, Egypt
}

\section{Key Words}

Taurine $\cdot$ Cafeteria diet $•$ Hepatokine $\cdot$ FGF-21 • SIRT1

\begin{abstract}
Background: Non-alcoholic fatty liver disease (NAFLD) is one of the alarmingly rising clinical problems in the $21^{\text {st }}$ century with no effective drug treatment until now. Taurine is an essential amino acid in humans that proved efficacy as a non-pharmacological therapy in a plethora of diseases; however, its impact on NAFLD remains elusive. The aim of the current study is to evaluate the protective mechanism of taurine in experimental steatohepatitis induced by junk food given as cafeteria-diet (CAF-D) in male albino rats. Methods: Forty adult male albino rats of local strain between 8-10 weeks old, weighing $150 \pm 20 \mathrm{~g}$, were divided into four equal groups: Group I (control group), Group II (Taurine group), Group III (CAF-D for 12 weeks) and Group IV (CAF-D + Taurine). CAF-D was given in addition to the standard chow for 12 weeks, where each rat was given one piece of beef burger fried in $15 \mathrm{~g}$ of sunflower oil, one teaspoonful of mayonnaise, and one piece of petit pan bread, weighing $60 \mathrm{~g} /$ piece. In the serum, liver function tests; ALT, AST, ALP, GGT and the lipid profile; TG, TC, HDL-C added to reduced glutathione (GSH) were assessed colorimetrically, while fibroblast growth factor (FGF)-21, adiponectin \& interleukin (IL)-6 via ELISA. The same technique was used for the assays of the hepatic levels of FGF-21, silent information regulator (SIRT1), malondialdehyde (MDA),IL-10, tumor necrosis factor- $\alpha$ (TNF- $\alpha$ ) as well as the apoptotic markers; caspase- 3 and B-cell lymphoma (Bcl-2). Results: The cafeteria-diet induced steatohepatitis was reflected by significantly increased body and liver weight gain, elevation of liver enzymes; ALT, AST, ALP and GGT added to the dyslipidemic panel, presented as increased TC, TG, LDL-C and decreased $\mathrm{HDL}-\mathrm{C}$ levels. The steatosis-induced inflammatory milieu, marked by elevated serum levels of FGF-21, IL-6, hepatic TNF- $\alpha$, as well as reduced IL-10 and adiponectin, was associated with steatosis- induced hepatic oxidative stress, reflected by increased hepatic MDA and decreased GSH levels, along with stimulated caspase-3 and decline in BcL-2 hepatic levels. These pathological disturbances were significantly ameliorated by taurine supplementation and evidenced histopathologically. The cross talk between hepatic FGF-21 and SIRT1 and their association to the induced perturbations are novel findings in this study. Taurine's efficacy in


restoration of hepatic structure and function is partially via the increase in SIRT1 and associated reduction of FGF-21. Conclusion: The findings of the current study prove the protective role of taurine in NAFLD via a novel role in the amelioration of FGF-21/ SIRT1 axis, which could be considered a new therapeutic target.

\section{Introduction}

Obesity is a major health problem and its incidence is increasing at an alarming rate not only in adults, but also among children and adolescents. The rising prevalence of obesity is attributed to changes in dietary habits with increased consumption of palatable junk food [1]. A "cafeteria" diet is a self-selected high-fat diet, providing an excess of energy, which proved to induce obesity. Excessive dietary lipid intake hampers glucose utilization, hence eliciting insulin resistance and abnormal postprandial lipid biotransformation [2]. Obesity predisposes to a variety of liver pathologies known as nonalcoholic fatty liver disease (NAFLD). It is not a homogeneous disease entity; depending on the severity of the pathological process, but may present as an isolated fatty degeneration (simple hepatic steatosis), nonalcoholic steatohepatitis (NASH), or lead to liver cirrhosis and hepatocellular carcinoma (HCC) $[3,4]$.

The development of hepatosteatosis arises as a result of an imbalance between triglyceride deposition and removal. Epidemiologically, NAFLD is associated with type 2 diabetes, suggesting that hepatosteatosis and the development of insulin resistance are causally linked and play a central role in the metabolic syndrome [5]. Molecular mechanisms responsible for the accumulation of fat in the liver are not fully understood; however, certain cytokines derived from inflammation sites, particularly from extrahepatic adipose tissues, can trigger this process. More prominently, insulin resistance is emerging at a core phase for the immense metabolic dysregulations of NAFLD that instigate and exacerbate hepatic steatosis. Pathological features of NASH include simple hepatic steatosis and, more characteristically, liver cell damage and accompanying inflammation and/or fibrosis. Recently, a plethora of pathogenic mechanisms have been determined for the evolution from simple steatosis to NASH, such as lipotoxicity, oxidative stress-induced mitochondrial dysfunction, as well as endoplasmic reticulum stress [6]. The "two-hit" hypothesis has been proposed to explain NAFLD pathogenesis with steatosis representing the "first hit", which increases the vulnerability of the liver to various "second hits" that in turn lead to inflammation, fibrosis and cellular death. Notably, oxidative stress is considered one such second hit, added to the inflammatory response, including the production of numerous proinflammatory molecules and adipokines; all together have key roles in the initiation and progression of the disease [7]. The current understanding of "the multiple parallel hypothesis" refers to NAFLD as a systemic, multifactorial disease involving multiple organs, such as adipose tissue, muscle and the intestine, as well as organelles such the endoplasmic reticulum and the mitochondria [8].

Fibroblast growth factor-21 (FGF-21) is a metabolic hormone with multiple metabolic functions. It belongs to the FGF superfamily that is involved in cell proliferation and differentiation, neural development, angiogenesis, and metabolism. Physiologically, FGF-21 is reported to be expressed from tissues involved in metabolism such as the liver, adipose tissues, skeletal muscle, and pancreas [9]. Secreted FGF-21 could regulate whole-body metabolism via its impact on the function of several tissues including brain and adipose tissues in an endocrine manner [10]. In white adipocytes, FGF-21 stimulates glucose uptake in an insulin-independent manner, modulates lipolysis and enhances mitochondrial oxidative capacity, and potentiates PPAR $\gamma$ activity [11]. There is also good evidence that FGF-21 is involved in the thermogenic functions of brown adipocytes [12, 13]. Notably, starvation is reported to increase hepatic expression of FGF-21, which then acts centrally to increase hepatic gluconeogenesis and fatty acid oxidation [14]. However, in obesity, fatty liver and high fat diet-induced metabolic pathologies, FGF-21 is expected to show different metabolic actions. 
Sirtuins type 1 (SIRT1), an important histone deacetylase, is a potential molecule and main determinant of whole body homeostasis in mammals by regulating many transcriptional regulators in metabolic tissues such as liver, skeletal muscle and adipose tissues [15]. SIRT1 and its activators play a key role in lipid and glucose homeostasis and insulin sensitivity via regulating mitochondrial biogenesis and $\beta$-oxidation, and improving anti-inflammatory activities. Previous studies indicated that the activation of SIRT1 in the liver protects against high-fat diet (HFD)-induced metabolic damage $[15,16]$, and that the regulation of hepatic lipid metabolism is postulated by SIRT1 deacetylation of sterol regulatory element-binding protein (SREBP)-1c, thus decreasing its expression $[17,18]$. Despite the high prevalence of NAFLD, an approved standardized treatment is still lacking, contributing to a serious challenge to health care systems. Diet and lifestyle modification remain the major non-pharmacologic therapy of NAFLD for the purpose of weight loss [19]. Insulin receptor sensitizing agents and antioxidants have been tested for the treatment of NAFLD, though their clinical efficacy and safety remain to be established [20]. Thus, it is necessary to explore novel therapeutics with high efficacy and fewer side effects. Amino acids have been recognized as important signaling mediators in different cellular functions. Taurine is a sulfur-including amino acid that is present in mammalian tissues in minute levels. It is endogenously synthesized from methionine and cysteine metabolism and is also provided in diets comprising fish and shellfish. Taurine is involved in various physiological functions, including bile salt conjugation, calcium homeostasis, osmoregulation, membrane stabilization, added to its anti-oxidant and immunomodulatory effects [21]. Global epidemiological studies revealed that people who consume a lot of sea food, containing taurine in abundance, have a lower risk of developing metabolic diseases such as obesity, diabetes, dyslipidemia, and hypertension [22]. To this end, the present study aims to examine the potential protective effects of taurine supplementation in CAF-D induced steatohepatitis in adult male albino rats, moreover, to determine the involved mechanisms.

\section{Material and Methods}

The composition of commercial rat chow diet (balanced diet) included $23.5 \%$ protein, $48.8 \%$ carbohydrate, $5 \%$ lipid, $12 \%$ water, $5 \%$ ash, $5 \%$ cellulose, and a $0.7 \%$ mixture of vitamins and minerals. The diet was designed at the National Research Institute for Nutrition, Giza, Egypt, according to the National Nutrition Database for Standard Reference. The diet was purchased from EL Nasr Pharmaceutical Chem. Co., New Maadi, Cairo, Egypt. The cafeteria diet (CAF) is also known as" Junk food" diet model:" JF" diet is a well-established model to study obesity; it is a highly palatable hyperlipidemic diet that mimics Western diet, inducing voluntary hyperphagia and rapid weight gain in rodents [2, 23]. CAF diet was composed of beef burger, bread and mayonnaise (purchased from local commercial sources). Each piece of beef burger weighed $150 \mathrm{~g}$ before cooking and looses between 15 and $20 \mathrm{~g}$ after cooking, resulting in an average new weight of 130-135g/piece. Each 100g of uncooked beef burger was composed of $18 \%$ protein, $17.7 \%$ total fat, $6.12 \%$ total saturated fatty acid, $45 \%$ carbohydrates, $12.3 \%$ water, and $0.8 \%$ vitamin mixture, according to the nutritive values recorded on the package. As regards mayonnaise, each teaspoonful gives 110 calories and is composed of $12 \mathrm{~g}$ fat, $2 \mathrm{~g}$ saturated fat, $10 \mathrm{~g}$ cholesterol, and $75 \mathrm{mg}$ sodium, according to the nutritive values recorded on the package [24].

Taurine (2-amino ethane sulfonic acid) was supplied by GALL PHARMA, Austria Pharmaceutical formed as taurine $500 \mathrm{mg} \mathrm{GPH}$ capsules.

\section{Experimental animals and design}

Forty adult male albino rats (8-10 weeks old, weighing 130-150 g) were purchased from the Nile Pharmaceuticals Company (Cairo, Egypt). They were housed in laboratory standard cages (2 rats /25X30X30 $\mathrm{cm}$ cage), under specific pathogen-free conditions in facilities maintained at controlled room temperature with a natural light/dark cycle. All animals had free access to chow diet and water ad libitum and were acclimated for one week prior to initiation of the experiment in the laboratory of Physiology, Faculty of Medicine, AI-Azhar University. All procedures were approved by the Animal Care Committee and the rules and principles of laboratory animal care were followed, as per the specific institutional guidelines. 
The rats were divided into 4 equal groups (10 rats/group). Group I (Control): rats were maintained on the standard diet for 12 weeks and served as control group. Groups II (Control/Tau): rats treated orally with taurine, freshly prepared by dissolving $500 \mathrm{mg} / \mathrm{kg} / \mathrm{b} . \mathrm{w} /$ day in distilled water [25], administrated by gastric gavage tube for 12 weeks. Groups III (CAF-D): rats fed a cafeteria diet in addition to the standard chow for 12 weeks. Each rat was given one piece of beef burger fried in $15 \mathrm{~g}$ of sunflower oil, one teaspoonful of mayonnaise, and one piece of petit pan bread, weighing 60g/ piece [26]. Group IV (CAF-D/Tau): rats were given taurine at a daily dose of $500 \mathrm{mg} / \mathrm{kg} /$ day dissolved in water by means of a gastric tube in addition to the 'junk food' as the same as group III for 12 weeks. The animal diet was introduced daily at 10 a.m. and the left over was not removed to ensure complete consumption by all rats.

Estimation of body weight and liver weight. Animals were regularly weighed and at the end of experiment they were sacrificed and their livers were quickly removed and weighed.

Serum biochemical analysis. At the end of the experiment, after overnight fasting, rats were anesthetized in the morning, and blood samples were collected from retro-orbital venous plexus by capillary tubes under light pentobarbitone anesthesia. The blood was then centrifuged at $3000 \mathrm{rpm}$ for 15 minutes for serum collection. Serum was separated in aliquots in Eppendorf tubes and stored frozen at $-80^{\circ} \mathrm{C}$ until analysis.

Assessment of hepatic enzymes. Serum alanine transaminase (ALT), aspartate transaminase (AST), alkaline phosphatase (ALP) and gamma glutamyl transferase (GGT) were calorimetrically determined as described by Ochei and Kolhackar [27] using commercial kits obtained from" Diamond Diagnostičs, Egypt.

Lipid profile. Serum total cholesterol (TC) and triglycerides (TG) were estimated as described by Sharma et al. [28]. Serum high density lipoprotein cholesterol (HDL-C) was measured according to the method described by Grillo et al. [29], while low density lipoprotein cholesterol (LDL-C) according to Friedewald et al. [30] as follows: LDL-C $=[\mathrm{TC}]-[\mathrm{HDL}-\mathrm{C}]-[\mathrm{TG} \backslash 5]$.

Glycemic Indices. Serum glucose level was assessed according to Tinder (1969) [31] using glucose enzymatic (GOD-PAP)-liquizyme Kits (Biotechnology, Egypt). Serum insulin was determined by a solid phase enzyme amplified sensitivity immunoassay according to Starr et al. [32] using KAP1251-INSEASIA (Enzyme Amplified Sensitivity Immunoassay) Kits (BioSource Europe S.A., Belgium). Estimation the Homeostasis Model of Assessment of Insulin Resistance was performed according to Matthews et al. (1985), where HOMA-IR= fasting insulin $(\mu \mathrm{U} / \mathrm{ml}) \times$ fasting blood glucose $(\mathrm{mg} / \mathrm{dl}) / 405$ [33].

Assessment of serum hepatokine and adipokine markers. Serum Fibroblast Growth Factor-21 (FGF21), IL-6 and adiponectin concentration were measured by ELISA using a commercially available kits according to the manufacturer's instructions [34]. FGF-21 was purchased from MyBioSource, Inc., San Diego, California, USA and adiponectin from Assaypro, Saint Charles, Missouri, USA). Serum IL-6 level was measured by commercial ELISA kits (Ray Bio® Rat, Ray Biotech, Norcross, GA, USA).

\section{Preparation of liver tissue homogenate}

The livers of the sacrificed albino rats were excised and the liver samples were cut into small pieces ( $1 \mathrm{~g}$ or $\mathrm{gm}$ ), minced and then homogenized in $1 \mathrm{ml}$ lysis buffer [ $20 \mathrm{mM}$ HEPES ( $\mathrm{pH} 7.5$ ), $150 \mathrm{mM} \mathrm{NaCl}, 1 \% \mathrm{NP}$ $40,0.1 \%$ SDS, $1 \mathrm{mM}$ EDTA, and $1.0 \mathrm{mM}$ DTT ] added to protease inhibitors (2 $\mu \mathrm{g}$ each of aprotinin, leupeptin, pepstatin A, and $0.5 \mathrm{mM}$ phenylmethylsulfonyl fluoride) then incubated on ice for $30 \mathrm{~min}$, and centrifuged at $10,000 \mathrm{xg}$ at $4^{\circ} \mathrm{C}$ for $20 \mathrm{~min}$ [35]. The resulting supernatant [cell lysate] was separated and stored at $-70^{\circ} \mathrm{C}$ until used for further analysis.

Assessment of liver oxidative stress profile. Malondialdehyde (MDA) level in the liver homogenate was assayed for products of lipid peroxidation by monitoring thiobarbituric acid reactive substance formation [36] using the colorimetric method. On the other hand, the method of GSH is based on the reduction of 5,5 dithiobis (2-nitrobenzoic acid) (DTNB) with reduced glutathione (GSH) to produce a yellow compound. The reduced chromogen is directly proportional to GSH concentration and its absorbance can be measured at $405 \mathrm{~nm}$. Both oxidative stress indicators, MDA and GSH, were assayed using a commercial kit (Biodiagnostic, Egypt).

Assessment of inflammatory cytokines. Both interleukin-10 (IL-10) and tumor necrosis factor-alpha (TNF- $\alpha$ ) in liver homogenate were detected by quantitative enzyme-linked immunosorbent assay (ELISA) using ELISA commercial kits (Quantikine, R\&D Systems, Minneapolis, USA) according to the manufacturer's instructions and according to Smith (1994) for TNF- $\alpha$ [37], while IL-10 was according to Croft et al. (2012) [38].

\section{KARGER}


Abd Elwahab et al.: Role of SIRT1/ FGF-21 in Hepatoprotective Effect of Taurine in Experimental NAFLD

Assessment of apoptosis in the liver. Bcl-2 protein content and Caspase-3 activity were measured in liver lysates by employing the quantitative sandwich enzyme immunoassay technique using ELISA kits supplied by Uscn Life Science Inc.(NYC,USA) and Quantikine (R\&D Systems, Minneapolis, USA); respectively [39]. The procedure was performed according to instructions of manufacturer.

Hepatokine marker in liver homogenate. Hepatic Fibroblast Growth Factor-21 (FGF-21) and SIRT1 concentration was measured by ELISA using a commercially available kit (My Bio Source, Inc., California, USA) according to the manufacturer's instructions.

\section{Histopathological Study}

Samples of liver tissues from each animal were fixed in $10 \%$ neutral buffered formalin for $24 \mathrm{~h}$. Washing was done with tap water and then serial dilutions of ethyl alcohol were used for dehydration. Specimens were cleared in xylene and embedded in paraffin at $56^{\circ} \mathrm{C}$ in hot air oven for $24 \mathrm{~h}$. Paraffin wax tissue blocks were prepared for sectioning at $4 \mu \mathrm{m}$ thicknesses by sledge microtone. The obtained tissue sections were collected on glass slides, deparaffinized, and stained with hematoxylin and eosin stain and Masson trichromate stain. Prepared slides were examined under a light electric microscope by a pathologist unaware of the treatment protocol.

\section{Statistical analysis}

All results were expressed as means \pm SD. Differences between groups were assessed by one-way analysis of variance (ANOVA), followed by a Bonferroni post hoc multiple comparison test. The association between the parameters was determined using the Pearson's correlation coefficient. A p value $<0.05$ was considered statistically significant. Statistical analysis was performed using the SPSS version 20.0 statistical software package (SPSS Inc., Chicago, IL).

\section{Results}

Since there was no difference between control group receiving taurine (Control/ Tau) and control group, only those for control are utilized in the current study for statistical comparison.

Effect of taurine on body and liver weight, serum lipid profile, liver function tests and glycemic indices

CAF-D induced a robust increase in body and liver weights $(1.3,2.25$ fold; respectively); effects that were significantly ameliorated by taurine administration by $14 \%$ and $30.5 \%$; respectively. Furthermore, the induced dyslipidemia was presented by a significant elevation of TG, TC, LDL-C (2.72-, 1.5-, 2.2-fold; respectively) and decrease in HDL-C by 37.7\%. These effects were associated with elevation of liver function tests; ALT, AST, ALP and GGT $(2.5,2.3$, 2, 2.1 fold; respectively) and perturbation of the glycemic indices; fasting glucose, insulin and HOMA-IR; by 1.3-, 1.6-, 2.4-fold; respectively. Notably, administration of taurine successfully ameliorated the aforementioned deleterious effects, as illustrated in Table1.

Table 1. Effect of taurine on physiological and serum laboratory indices in adult male albino rats with steatohepatitis. Values are presented as means \pm SDM. ( $*$ ) Value significantly different vs. control group or ( \#)from CAF-D group, at $\mathrm{P}<0.05$

\begin{tabular}{lllll}
\hline Groups/ Parameters & Control & Control/Tau & CAF-D & CAF-D +Tau \\
\hline -Final body weight(g) & $221.66 \pm 4.7$ & $219.0 \pm 4.1$ & $295.16 \pm 6.2^{*}$ & $254.16 \pm 6.2^{* \#}$ \\
- Liver weight (g) & $4.05 \pm 0.5$ & $3.98 \pm 0.3$ & $9.0 \pm 0.39^{*}$ & $6.25 \pm 0.6^{* \#}$ \\
Serum Lipid Profile & & & & \\
TG (mg/dl) & $73.71 \pm 5.3$ & $73.61 \pm 6.6$ & $200.58 \pm 13.5^{*}$ & $135.76 \pm 22^{* \#}$ \\
TC (mg/dl) & $90.32 \pm 9$ & $89.67 \pm 7$ & $131.79 \pm 17.3^{*}$ & $107.98 \pm 10.69$ \# \\
HDL-C(mg/dl) & $46.94 \pm 9.5$ & $49.64 \pm 6.5$ & $29.21 \pm 7.87^{*}$ & $43.26 \pm 9.83^{\#}$ \\
LDL-C(mg/dl) & $28.6 \pm 6$ & $27.61 \pm 6.6$ & $62.46 \pm 16.86^{*}$ & $37.56 \pm 11$ \# \\
Serum liver enzymes & & & & \\
ALT (IU/L) & $35.65 \pm 7.11$ & $35.68 \pm 6.79$ & $89.71 \pm 13.64^{*}$ & $43.92 \pm 6.3^{\#}$ \\
AST(IU/L) & $36.71 \pm 5.87$ & $36.1 \pm 4.77$ & $83.75 \pm 8.56^{*}$ & $40.74 \pm 6.54^{\#}$ \\
ALP (IU/L) & $82.31 \pm 2.27$ & $81.5 \pm 4.36$ & $165.57 \pm 4.33^{*}$ & $101.55 \pm 3.36^{* \#}$ \\
GGT (IU/L) & $19 \pm 8.9$ & $22.3 \pm 3.8$ & $61.3 \pm 3.7^{*}$ & $27.8 \pm 3.8^{* \#}$ \\
& & & & \\
Glycemic indices & & & & \\
Fasting glucose(mg/dl) & $80.68 \pm 4.92$ & $79.48 \pm 4.96$ & $104.14 \pm 4.81^{*}$ & $86.98 \pm 5.26^{\#}$ \\
Fasting insulin ( $\mu \mathrm{U} / \mathrm{ml})$ & $30.55 \pm 4.8$ & $28.84 \pm 3.9$ & $50.34 \pm 7.4^{*}$ & $36.88 \pm 5.54^{\#}$ \\
HOMA-IR & $5.46 \pm 0.32$ & $4.99 \pm 0.78$ & $12.97 \pm 2.3^{*}$ & $7.91 \pm 1.51^{* \#}$ \\
Serum Adiponectin (ng/ml) & $59.58 \pm 1.68$ & $62.32 \pm 4.97$ & $30.8 \pm 2.84^{*}$ & $52.09 \pm 3.24^{* \#}$ \\
Serum IL-6 (pg/ml) & $2.2 \pm 1.2$ & $1.9 \pm 1.1$ & $8.4 \pm 2.2^{*}$ & $4.3 \pm 2.1^{* \#}$ \\
Serum FGF-21 (pg/ml) & $75.23 \pm 2.7$ & $73.6 \pm 2.5$ & $157.7 \pm 6.4^{*}$ & $93.05 \pm 3.63^{* \#}$ \\
\hline
\end{tabular}




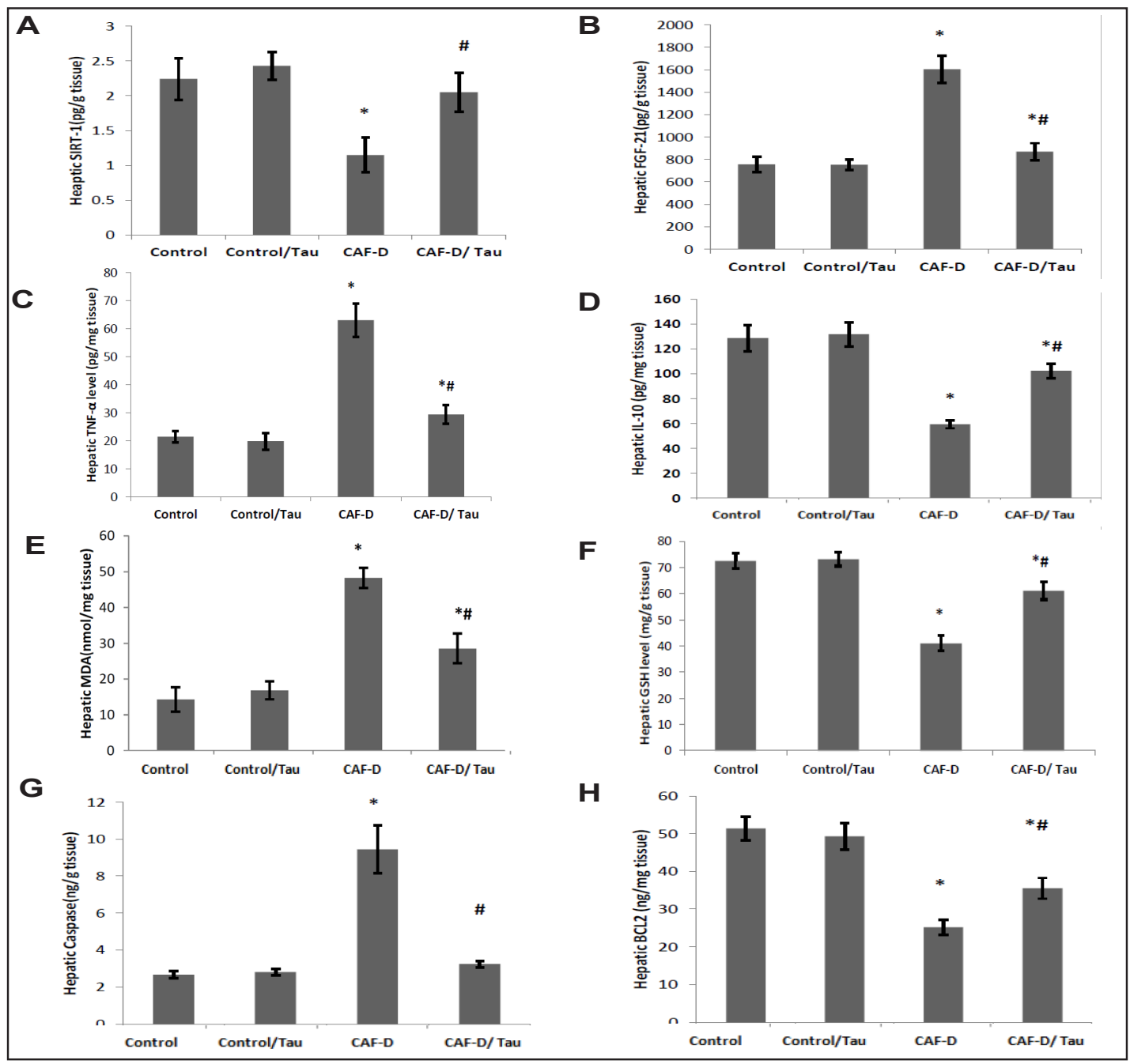

Fig. 1. Effect of taurine on hepatic levels of SIRT1(Fig.1A), FGF-21 (Fig.1B), TNF- $\alpha$ (Fig.1C), IL-10 (Fig. 1D), MDA(Fig. 1E), GSH (Fig. 1F), casapase-3 (Fig. 1G) and BcL-2 (Fig. 1H) in different experimental groups. Data represent the means $\pm \mathrm{SDM},\left(^{*}, \#\right)$ compared with control, CAF-D, respectively, at $\mathrm{P}<0.05$, using one-way ANOVA followed by Bonferroni post hoc multiple comparison test.

Effect of taurine on CAF-D - induced NAFLD changes in adiponectin, IL-6, FGF-21 and SIRT1 levels

Rats fed CAF-D showed marked decrease in serum adiponectin (-48.3\%) together with elevation of serum levels of IL-6 and FGF-21 ( 2 fold, 4 fold their control levels; respectively). Conversely, taurine administration concomitantly with CAF-D decreased both serum levels of FGF-21 and IL-6 (47\%, 50\%; respectively), while increased serum adiponectin by 1.7 fold; data illustrated in Table 1. Concerning the hepatic levels of SIRT1 (Fig.1A) and FGF21 (Fig. 1B), they showed opposite patterns. While hepatic level of FGF-21, in the CAF-D group, showed a two-fold elevation of the control level, administration of taurine restored normal level via a $45.7 \%$ significant reduction. Conversely, hepatic SIRT1 was significantly decreased (50\%) upon experimental induction of NAFLD model, and taurine significantly ameliorated this effect by 1.74 fold increase in its level.

Effect of taurine on CAF-D -induced hepatic inflammatory and oxidative stress markers

CAF-D for 12 weeks showed an evident disturbance in the hepatic pro-/ antiinflammtory equilibrium, by causing a prominent increase in levels of hepatic proinflammatory marker, TNF- $\alpha$ (2.9 fold; Fig. 1C) and decrease in anti-inflammatory cytokine, IL-10, by 53.8\% (Fig. 
Fig. 2. (A-G) Microscopic examination of hepatic tissues of the assessed groups shows the following results, (A) A photomicrograph from a liver of control rat showing normal hepatocyte with euchromic nuclei with no fibrosis around the central vein (blue arrow) and portal tracts (black arrow). X 200 $\mathrm{H} \& \mathrm{E}$. (B) A photomicrograph from a liver of control rat showing normal distribution of the collagen fibers in the central vein (blue arrow) and the portal tracts (black arrows) X 100 masson-trichrome. (C)A photomicrograph from a liver of a rat in CAF-D group showing thick wall of hepatic artery, fatty degeneration (blue arrow), with inflammatory cell infiltration (black arrow) as lymphocytes and mononuclear cells with focal areas of degeneration, congestion and necrosis.(x $200 \mathrm{H} \& \mathrm{E}$ ). (D) A photomicrograph from a liver of a rat in CAF-D group showing excessive deposition of collagen fibers in the central vein and portal tracts X 100 masson trichrome. (E) A photomicrograph from a liver of taurine group (Control/Tau) showing the nearly normal architecture of hepatic lobule with no fibrosis around the portal tracts ( x $200 \mathrm{H} \mathrm{\&} \mathrm{E).}$ (F) Liver cell of taurine pretreated CAF-D group showing congested vessels, hepatocytes with pyknotic hepatic tissue with moderate distribution of the nuclei (blue arrow) and some fat globules (black arrow) and cellular infilteratin. (x $100 \mathrm{H} \&$

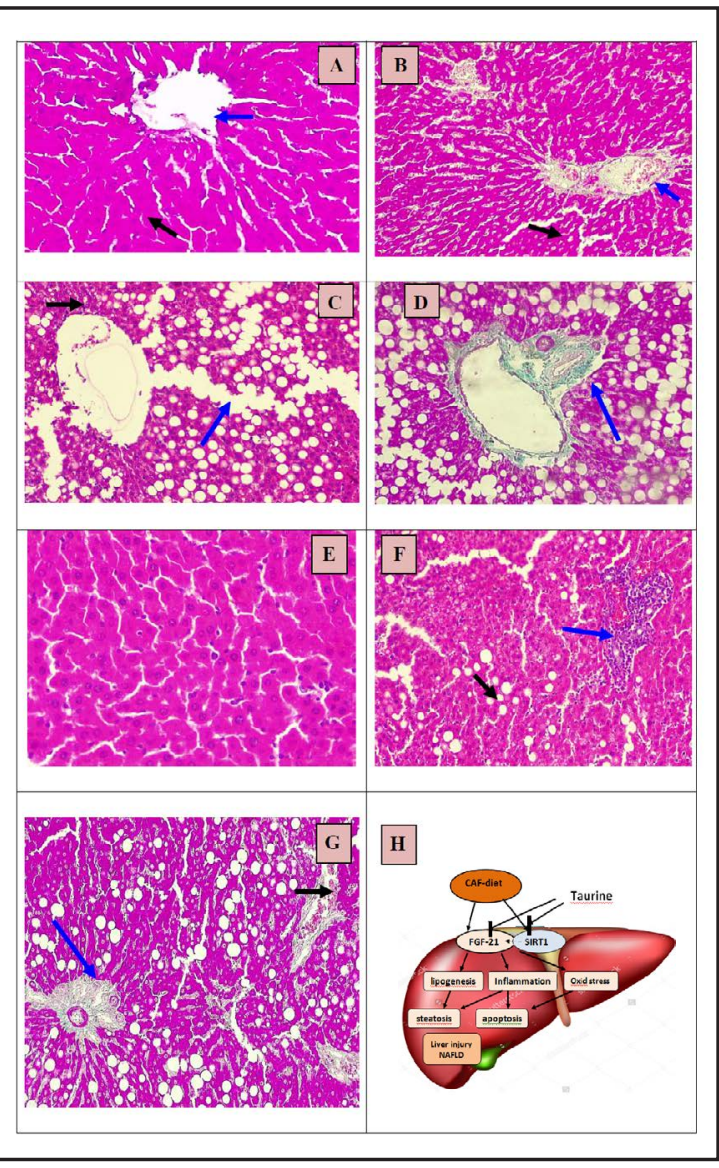
E). (G) Liver of taurine pretreated CAF-D group showing some hepatocytes with pyknotic nuclei and moderate distribution of the collagen fibers in the central vein (blue arrow) and the portal tracts (black arrows) (X100 masson trichrome). (H) Schematic illustration of mechanisms of CAF-D induced NAFLD and the hypothesized ameliorative effect of taurine. Habitual consumption of CAF-D stimulates hepatic SIRT1, which in turn promotes expression, production and release of hepatic FGF-21. Induction of hepatic and circulating levels of FGF-21 stimulates lipogenesis, oxidative stress and inflammation. Activation of SIRT1-FGF-21 cascade may represent a liver-adipose tissue axis that contributes to hepatic steatosis and apoptosis leading to NAFLD. Taurine proved to inhibit SIRT1-FGF-21 axis and prevent the deleterious sequelea.

1D). These effects were associated with an increase in hepatic MDA level (2.8 times; Fig. 1E) and decrease in hepatic antioxidant marker, GSH (3.5\%; Fig. 1F). On the other hand, animals given taurine showed a decline in TNF- $\alpha$ and MDA levels and increase in IL-10 and GSH levels.

\section{Effect of taurine on CAF-D -induced NAFLD on hepatic apoptotic markers}

CAF-D- induced NAFLD in rats produced significant apoptosis in hepatic tissue as manifested by increased caspase-3 (3.5 fold, Fig. 1G) and decreased BcL-2 (50\%; Fig. 1H), compared to their respective controls. On the other hand, taurine administration concomitantly with CAF-D decreased caspase- 3 activity by $51 \%$, as well increased BcL- 2 by 1.4 folds.

\section{Histopathological effect of taurine on CAF-D - induced NAFLD changes}

Microscopic examination of hepatic tissues of CAF-D supplemented group showed fatty degeneration with inflammatory cell infiltration of lymphocytes and mononuclear cells with focal areas of degeneration, congestion and necrosis. (Fig. 2C), and marked collagen fiber deposition around the central vein and portal tracts (Fig. 2D) compared to normal patterns (Fig. 2A and 2B). Taurine supplementation showed nearly normal hepatic architecture (Fig. 
2E). Taurine supplementation for twelve weeks with CAF-D- Table 2. Correlational analysis betintake resulted in ameliorative process of hepatic cells mor- ween serum and hepatic FGF-21, phologies in the form of marked reduction of the amount h-SIRT1 and the assessed parameof fat globules with decreased cellular infiltration (Fig. 2F). ters

Also, the liver cells showed some hepatocytes with pyknotic nuclei and moderate distribution of the collagen fibers in the central vein and the portal tracts (Fig. 2G).

\section{Correlation analysis}

The correlation analysis, illustrated in Table 2 , revealed significant association between serum vs. hepatic FGF-21 and the following markers; serum adiponectin $(r=-0.97,-$ 0.95; respectively), hepatic levels of TNF- $\alpha(r=0.97,0.97$; respectively), IL-10 ( $\mathrm{r}=-0.97,-0.96)$, GSH ( $\mathrm{r}=-0.97,-0.95)$; MDA ( $r=0.92,0.91)$, BcL2 $(r=-0.92,-0.88)$ and caspase-3 ( $r=0.98,0.99)$, all respective to serum vs hepatic FGF2 , at $\mathrm{p}<0.05$. The correlation between hepatic SIRT1 and assessed parameters revealed negative association with HOMA-IR $(r=-0.86)$, hepatic levels of FGF-21 $(r=-0.89)$, TNF- $\alpha(r=-0.87)$, MDA ( $r=-0.88)$ and caspase- $3(r=-0.88)$; whereas positive correlation with serum adiponectin $(\mathrm{r}=$ $0.88)$ and hepatic levels of IL-10 $(r=0.87)$, GSH $(r=0.87)$;

\begin{tabular}{lccc}
\hline & s-FGF- & h-FGF- & h-SIRT1 \\
\hline Serum levels & 21 & 21 & \\
TG & 0.92 & 0.89 & -0.83 \\
LDL-C & 0.81 & 0.89 & -0.69 \\
AST & 0.97 & 0.68 & -0.88 \\
ALT & 0.93 & 0.93 & -0.79 \\
HOMA-IR & -0.90 & 0.90 & -0.86 \\
Adiponectin & -0.97 & -0.95 & 0.88 \\
IL-6 & 0.95 & 0.94 & -0.86 \\
Hepatic levels & & & \\
h-FGF-21 & 0.99 & -------- & -0.89 \\
h-SIRT1 & -0.90 & -0.89 & $---1-$ \\
TNF- $\alpha$ & 0.97 & 0.97 & -0.87 \\
IL-10 & -0.97 & -0.96 & 0.87 \\
MDA & 0.92 & 0.91 & -0.88 \\
GSH & -0.97 & -0.95 & 0.87 \\
BcL-2 & -0.92 & -0.88 & 0.83 \\
Casapse-3 & 0.98 & 0.99 & -0.88 \\
\hline
\end{tabular}
and BcL2 $(r=0.83)$, all at $\mathrm{p}<0.05$.

\section{Discussion}

There is consensus that the pathogenesis of NAFLD is as a multi-hit process that includes lipotoxicity, oxidative challenge and endoplasmic reticulum (ER) stress [40]. In the current study, taurine administration inhibited the NAFLD multi-hit process, based on lipid profile optimization, improvement of glycemic indices, increased adiponectin level, mitigation of hepatic oxidative stress markers (through reduction of MDA), inflammation (IL-6\&TNF- $\alpha$ ), apoptosis (caspase-3), and enhanced hepatic GSH, IL-10 and Bcl-2 levels. The most important implication of the present study is the identification of novel cross-talk between nutrient sensor SIRT1 and FGF-21, in the current model of steatohepatitis, where SIRT1/ FGF-21 axis could represent a novel hepatocyte-derived endocrine signaling to potentially combat hepatic steatosis and obesity in humans. Several studies reported a positive association between SIRT1 and FGF-21 in starvation, feeding and obesity-related disorders, with the latter being the downstream regulator of the former. Interestingly, the assessment of hepatic levels of SIRT1 protein content, in the current CAF-D-induced NAFLD, revealed a marked decrease in its expression, with concomitant elevation in FGF-21; perturbations that were successfully normalized by taurine supplementation. The NAFLD-induced reduction of SIRT1 expression was previously evidenced in experimental and clinical studies. Deng et al. [41] reported a reduction of SIRT1 expression in rats with NAFLD induced by high-fat diets. In addition, Mariani et al. [42] observed that SIRT1 expression was significantly decreased in visceral adipose tissue of morbidly obese patients with severe steatosis, compared to patients with slight or moderate steatosis. Remarkably, the molecular mechanisms of the action of taurine in correction of NAFLD was reported in the study of Banks et al. [43], who explained that taurine increases the expression of SIRT1 in the liver which further stimulates the antioxidant capacity, by SOD induction and lowering the proinflammatory signals, TNF- $\alpha$ and interleukin 6 (IL-6). Moreover, taurine was reported to induce overexpression of SIRT1 in mice which provided protection against HFD induced hepatic steatosis through upregulation of fatty acid oxidation and downregulation of lipogenesis [15].

FGF-21, a hepatokine, has recently been shown to possess beneficial effects on lipid metabolism and hepatic steatosis [9]. In the present study, both serum and hepatic FGF-21 
levels showed marked increase in CAF-D supplemented group when compared to normal control rats. Notably, Hondares et al. [12] reported that exogenous administration of FGF21 increased browning of white adipose tissue, energy expenditure and glucose disposal. Additionally, FGF-21 regulates energy homeostasis in adipocytes through activation of AMPK and SIRT1, resulting in enhanced mitochondrial oxidative function. In alignment with our findings, several studies demonstrated that serum FGF-21 concentrations were significantly increased and associated with hepatic fat content especially in subjects with moderate hepatic steatosis $[13,44]$ and could, therefore, be used as useful circulating biomarker for predicting progression in NAFLD patients. This elevation of FGF-21 levels was consistent with the data of Wree et al. [45] who reported concomitant reduction of adiponectin. Moreover, they revealed that reduced adiponectin levels may establish proinflammatory changes, thus increasing vulnerability to lipotoxicity, exacerbating hepatocytes injury, factors that promote progression from simple steatosis to NASH and even advanced hepatic fibrosis. This is further confirmed by the current negative association between FGF-21 and adiponectin. Another explanation was reported by Yu et al. [46] that elevated serum FGF-21 is likely due to time-dependent expression of FGF-21 mRNA in human hepatocytes, which is more related to unsaturated fatty acids but is opposite to the glucometabolic pattern of insulin and glucose.

Several clinical studies explained that the increased level of FGF-21, in patients with a cluster of obesity-related disorders including nonalcoholic fatty liver disease, is considered a compensatory, adaptive response to the induced proinflammatory milieu [47]. This is further evidenced by the significant positive correlation between FGF-21 and TNF- $\alpha$ in the current study. Taurine supplementation, in the current study, induced significant decrease in both serum and hepatic FGF-21. We speculate that the improvement in steatosis mediated by taurine is the result of the reduced FGF-21 levels. Furthermore, Terashima et al. [48] reported that taurine supplementation ameliorated oxidative stress and fibrosis which caused correction of the pathogenesis of NAFLD. Collectively, these results indicate that the secretion of FGF-21, in the context of hepatic damage, may serve as an endogenous, autoregulatory, hepatoprotective signaling pathway against pro-oxidative damage.

The novelty of the current study lies in the new relation between FGF-21 and SIRT1 in this experimental model of NAFLD and the mechanism of taurine in correcting this pathogenesis. A plethora of studies addressed the controversial relationship between FGF21 and SIRT1, yet there no consensus about their causal relationship. A previous study of Li et al. reported that SIRT1 regulates FGF-21 expression in hepatocytes in vitro and in vivo; however, the relative contribution of FGF-21 to SIRT1's effects on overall hepatic metabolism in different hepatic disease states has not been investigated [49]. They characterized FGF-21 as a critical downstream regulator of SIRT1 that protects against hepatic steatosis, enhances expression of brown fat-like genes in white adipose tissue, and increases whole-body energy expenditure. This further supports the perception that SIRT1 induces gene and protein expression of FGF-21 in hepatocytes. The negative association between FGF-21 and SIRT1, added to CAF-D induced reduction in hepatic SIRT1 and concomitant elevation in hepatic and circulating FGF-21 levels are new findings in the current study. Similar findings, but in a heart failure model, were explained by Planavila et al. [50] who stated that SIRT1 activity can be modulated by the action of specific metabolic activators and mechanisms other than changes in its expression level. Thus, the up-regulation of FGF-21 in failing hearts may be mediated by post-transcriptional changes in SIRT1, although SIRT1 expression was induced in failing human hearts.

While most of the studies of NAFLD focus on SIRT1 and SIRT-3, the mitochondrial SIRT4 deserves more consideration. Tarantino et al. [51] showed low circulating levels of SIRT4 in obese patients with NAFLD concomitant with its reduced mitochondrial expression to increase the fat oxidative capacity together with the hepatic and muscular mitochondrial functions. Interestingly, SIRT4 controls the metabolism of free fatty acids (FFA) reducing their high circulating levels but increasing ROS production as well. 
At the metabolic levels, CAF-D for 12 weeks verified the reported pathogenic characteristics of NAFLD, including obesity, lipotoxicity, oxidative stress and apoptosis. The induced increase in mean final body and liver weights [24] was accompanied by overt dyslipidemia; both causing elevation of liver enzymes. Yang et al. [52] attributed this increase in the aforementioned weights first to the hyperphagia and consequently, high energy intake induced by adipocyte-derived leptin hormone secretion due to high fat diet intake. The second cause is the consumption of HFD contents of saturated fats, which are considered to be one of main contributors to overweight and obesity, because fat is digested into monoglycerides and fatty acids by lipase and the absorbed fat is hence accumulated in adipose tissue through excessive adipocyte differentiation.

Taurine with CAF-D supplementation for twelve weeks induced significant reduction in body and liver weights, ameliorated the dyslipidemia and the liver function tests. The reduction in body and liver weights was explained by Murakami et al. [21] who clarified that taurine conjugation with bile acid and the neutralization of hypochlorous acid produced by phagocytic cells in the body, generate taurine-conjugated bile acids and taurine chloramines (Tau-Cl), respectively. These products are also active and exhibit a variety of physiological activities, including anti-inflammatory and antioxidative effects, modulation of glucose, and lipid metabolism and reducing the body and liver weights. Moreover, the antiobesity effect of taurine was also explained by Tsuboyama-Kasaoka et al. [53], who clarified that dietary taurine prevents the high-fat diet-induced obesity with increased resting energy expenditure through its effect in decreasing the white fat and increasing the brown fat weight. PinaZentella et al. [54] reported that taurine enhances the rate of lipolysis in isolated adipocytes by stimulating protein kinase A (PKA) activity. The aforementioned events explain the logic sequelae of dyslipidemia in the current study, evidenced by marked elevation in the total cholesterol, triglycerides, LDL-C and reduction of serum HDL-C. NAFLD is reported to closely associate with risk factors such as dyslipidemia and increased hepatic contents [55, 56]. In this study, CAF-D caused significant elevation of serum ALT, AST, ALP and GGT indicating hepatic dysfunction, cellular leakage and loss of functional integrity of membrane hepatocytes with more specificity of ALT enzyme in liver injury assessment [57]. In addition, elevated serum levels of ALP and bilirubin give a clue of hepatobiliary injury, especially interruption of the flow of bile from the liver, i.e. cholestasis [58]. Taurine supplementation, in the present work, showed improvement of the induced dyslipidemia which was explained by the reported physiologic hypocholesterolemic and antiatherogenic effects in experimental animals [59]. Notably, Fukuda et al. [60] reported that the lowering effect of dietary taurine of serum cholesterol was partly attributable to a reduced secretion rate of cholesteryl ester by the liver, and that reductions in hepatic secretion rate and hepatic accumulation of cholesteryl ester were inversely related to increased ketone body production, an index of fatty acid oxidation. This pattern of changes suggested that dietary taurine influences the metabolic fate of fatty acids, either they undergo esterification or oxidation. Moreover, Lam et al. [61] stated that taurine increases the expression level of cholesterol 7a-hydroxylase (CYP7A1) mRNA in HepG2 cells, which decreases the cholesterol pool in liver causing some secondary changes, as enhancing the hepatic LDL- receptor activity and its binding of LDL-C. Other mechanisms have been reported such as the inhibition of very low-density lipoprotein (VLDL) secretion from the liver, the suppression of hepatic acyl-CoA: cholesterol acyltransferase (ACAT) activity, and the inhibition of the absorption of bile acid from the intestinal tract [62]. Plasma high-density lipoprotein (HDL) is considered as antioxidant protein and involved in antiatherogenic activities. Plasma HDL-C can reduce the level of nonesterified fatty acid hydroperoxides and participates in the antioxidant property of HDL-C [63]. The efficacy of taurine to cause amelioration in the lipid and metabolic profiles was reflected by a further correction in the ALT and AST levels, as corroborated by our histopathologic findings of liver tissues.

Insulin resistance (IR) is the key factor in the pathogenesis of NAFLD and is deeply entangled with the progression of fatty liver to advanced stages of fibrosis and cirrhosis [64]. During the course of NAFLD, in our rats, we found that fasting blood glucose and insulin

\section{KARGER}


levels were significantly higher in NAFLD, compared to control rats, associated with a higher degree of insulin resistance index (HOMA-IR). Moreover, Milnar et al. [64] and Stanhope \& Havel [65] referred this IR to that visceral adiposity, induced by HFD, that causes direct delivery of portal blood flow from visceral fat to the liver releasing FFAs. Increased amounts of FFAs is reported to directly affect insulin signaling, diminish glucose uptake in muscle, and induce gluconeogenesis in the liver. Another explanation was reported by El Mesallamy et al. [66] that HFD intake activates serine kinases coupled with inhibition of tyrosine phosphorylation of the insulin receptor which could result in IR. Changes in redox balance can activate certain stress induced serine kinases which can, in turn, decrease the extent of tyrosine phosphorylation, and is consistent with the attenuation of insulin action. All these changes were successfully ameliorated by taurine supplementation.

Adiponectin is a protective adipokine that antagonizes hepatic lipid accumulation in addition to insulin-sensitizing, anti-inflammatory and antifibrotic effects. Decreased adiponectin levels play a pivotal role in the pathogenesis of NAFLD [67]. This information supports our results that CAF-D induced significant reduction of serum adiponectin, which was corrected by taurine supplementation [68, 69]. Kim et al. [70] stated that taurine significantly increased serum adiponectin expression by inhibiting STAT-3 signaling in IL$1 \beta$-stimulated adipocytes, independent of MAPK signaling, suggesting that taurine plays a significant role in modulating the expression of adipokines under inflammatory conditions. Concomitant with reduced adiponectin in the current study, CAF-D induced an elevation of TNF- $\alpha$ with significant reduction of IL-10. Intriguingly, taurine supplementation effectively ameliorated TNF- $\alpha$ and IL-10 levels, compared with CAF-D group. As the oxidative stress is tightly linked to mediators of inflammation, both playing a vital role in the pathogenesis of NAFLD, the proinflammatory cytokines, TNF- $\alpha$ and IL-6, were secreted from various cells/ tissues including adipose tissue. Various clinical studies have suggested that drugs that reduce the levels of inflammatory markers [71], and elevate serum adiponectin level may provide a better treatment strategy for NAFLD [72]. On the other hand, IL-10 is an important antiinflammatory cytokine that is known to be an important factor in maintaining homeostasis of overall immune responses [73]. Su et al. [74] observed that administration of taurine in cardiac patients induces a potent anti-inflammatory response in the form of decrease in serum IL-6 and an increase in serum IL-10 with significant improvement in arrhythmias. Another study of Yang et al. highlighted the ability of taurine to reduce epidural fibrosis in rat models after laminectomy via downregulating early growth response protein 1 (EGR1), an enhancer of fibrosis, both in vivo and in vitro [75].

CAF-D supplementation for 12 weeks induced significant oxidative stress in the form of elevated MDA and reduced GSH [76, 77]. Nobili et al. [78] reported that oxidative stress is one of the main causes of NAFLD, which is further exacerbated with steatohepatitis and represented with elevated malondialdehyde (MDA) and reduced glutathione (GSH), the biomarkers of oxidative stress. Taurine is known to improve cellular antioxidant defense system, stabilize biomembranes and reduce in vivo lipid peroxidation (LPO), thus preventing apoptosis and necrotic cell death [79]. Parvez et al. [80] attributed the anti-oxidant property of taurine to the inhibitory effect of mitochondrial superoxide generation, thus suggesting that the anti-oxidant properties of taurine are mediated by mitochondrial pathways. The molecular mechanisms involved in this process have been evaluated in in vitro studies of Jong et al. [81] by depleting mitochondrial taurine levels. The depletion of taurine in mitochondria causes a decline in the biosynthesis of mitochondrial-encoded proteins ND5 and ND6, which leads to the impairment of complex I and III activities and results in the excessive production of superoxide. Therefore, taurine functions as an indirect anti-oxidant which may help to prevent NAFLD.

The performed experimental NAFLD induced activation of caspase-3 which indicates an increase in the apoptotic activity in the liver tissue. Bcl-2 is a prosurvival multidomain protein that regulates apoptosis by preventing the release of proapoptogenic factors from the mitochondria (e.g.cytochrome $c$ ) and subsequentcaspase activation [82]. In our study, the antiapoptotic protein Bcl-2 level was significantly reduced with CAF-D intake; this dysregulation 
of the fine-tuned apoptotic pathway is considered one of the mechanisms of CAF-D induced injury of the liver which might also explain the elevated liver enzymes. Moreover, Tarantino et al. reinforced our results, in his clinical study, that showed significantly lower serum Bcl2 concentrations in nonalcoholic steatohepatitis (NASH) subjects, compared to fatty liver patients. Insulin resistance and GGT activity were also considerably higher in NASH subjects, concomitant with significantly more numerous apoptotic hepatocytes [83]. Several in vitro studies demonstrated that ER stress is the mediator of saturated FFA-induced apoptosis. As intracellular FFA are trafficked to and esterified within the endoplasmic reticulum (ER), it is not surprising, that the inundation of the liver with FFA (termed lipoapoptosis) disturbs ER function resulting in an ER stress response through depleting ER-calcium stores in liver cells [84]. JNK activation can be linked to dysregulation of this core cell death machinery, promoting cell death, as it induces mitochondrial dysfunction and apoptosis via BCL2-associated Xprotein (Bax). Apoptotic signaling is activated when Bax migrates to the mitochondrial surface, promoting cyt-c and apoptosis inducing factor (AIF) release from the mitochondria to the cytosol, cleaving procaspase- 9 to caspase- 9 , and activating caspase- 3 to promote cell death [85]. Additionally, Guha et al. [86] demonstrated that apoptotic stimuli such as GSH depletion promote the translocation of mitochondrial Bax, which is a proapoptotic Bcl-2 family of proteins that decreases the mitochondrial membrane potential (MMP) and promotes the generation of ROS.

The protective role of taurine is highlighted in the adjustment of caspase- 3 activities and Bcl-2 level towards the control values in order to properly control the proliferation and differentiation of liver cells. This anti-apoptotic effect of taurine is in consistence with Watanabe et al. [87] who documented an anti-apoptotic effect of oyster extract that is rich in taurine in NASH-model mice, and Gentile et al. [88] who found that taurine significantly mitigated palmitate-mediated caspase- 3 activity, cell death, ER stress, and oxidative stress in H4IIE liver cells and primary hepatocytes.

\section{Conclusion}

Taurine administration inhibited the NAFLD multi-hit process, based on reduced lipotoxicity of CAF-D by lipid profile optimization, improvement of glycemic indices, increased adiponectin level, mitigating hepatic markers of oxidative stress through reduction of MDA, inflammatory marker (TNF- $\alpha$ ) and apoptosis (caspase-3) as well as enhanced hepatic GSH, IL-10 and Bcl-2 levels, compared with CAF-D group. Intriguingly, the negative association between hepatic FGF-21 and SIRT1 in the NAFLD model is a novel finding in this study. This SIRT1/FGF-21 axis represents a novel hepatocyte-derived endocrine signaling to potentially combat hepatic steatosis and obesity in humans. Taurine's efficacy in restoration of hepatic structure [which was evidenced histologically] and function is partially due to its reduction of FGF-21 via increase in SIRT1 hepatic level. These findings indicate that taurine has antioxidant and anti-apoptotic effects on NAFLD model cells and may thus exert protective effects against NAFLD. Additionally, taurine has a number of advantages as a potential protection for NAFLD in that it is inexpensive, nontoxic, and can be administered orally. Our study supports the rationale for targeting FGF-21/ SIRT1 axis as a novel complementary approach to treat hepatic steatosis.

\section{Limitation of the study}

As FGF-21 has potential pleiotropic actions in hepatocytes and adipocytes, the authors acknowledge the inability to assess the levels of SIRT1 and FGF-21 in the adipocytes and relate them to the assessed hepatic levels. Moreover, the autocrine/paracrine actions of FGF-21 could be assessed in white adipose tissue and in brown adipocytes as well to delineate its thermogenic activity. Considering the reported strong involvement of the mitochondria in the pathogenesis of NAFLD, theauthors would like to acknowledge theinability to isolate the mitochondrial hepatocytes to determine SIRT-4 expression, which is another important sirtuin in the pathogenesis of NAFLD. 


\section{Cellular Physiology Cell Physiol Biochem 2017;43:644-659

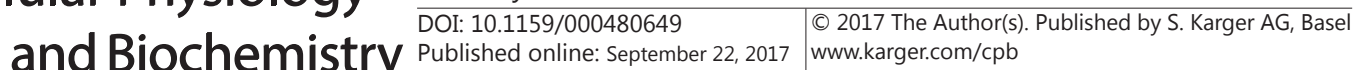

Abd Elwahab et al.: Role of SIRT1/ FGF-21 in Hepatoprotective Effect of Taurine in Experimental NAFLD

\section{Disclosure Statement}

The authors declare having no funding sources for this research and no conflict of interest.

\section{References}

1 Bayol SA, Simbi BH, Fowkes RC, Stickland NC: A Maternal “Junk Food” Diet in Pregnancy and Lactation Promotes Nonalcoholic Fatty Liver Disease in Rat Offspring. Endocrinol 2010;151:1451-1461.

-2 Sabater D, Agnelli S, Arriaran S, Romero M, Fernandez-Lopez JA, Alemany M, Remesar X: Cafeteria diet induce changes in blood flow that are more related with heat dissipation than energy accretion. Peer J 2016;4,e2302:1-11.

-3 Sangi SMA: Potential therapeutic agents for the treatment of fatty degeneration of liver and atheromatous plaques: an experimental study in rats. Pharmacogn Mag 2016;12:S414-S423.

4 Lebensztejn DM, Flisiak-Jackiewicz M, Białokoz-Kalinowska I, Bobrus-Chociej A, Kowalska I, Dariusz M: Hepatokines and non-alcoholic fatty liver disease. Acta Biochim Pol 2016;63:459-467.

5 Cohen JC, Horton JD, Hobbs HH: Human fatty liver disease: old questions and new insights. Sci 2011;332:1519-1523.

6 Yu J, Marsh S, Hu J, Feng W, Wu C: The pathogenesis of nonalcoholic fatty liver disease: interplay between diet, gut microbiota, and genetic background. Gastroenterol Res Pract 2016;1-13.

7 Wang H J, Gao B, Zakhari S, Nagy L E: Inflammation in alcoholic liver disease. Annu Rev Nutr 2012;32,343368.

8 Nassir F, Ibdah JA: Sirtuins and nonalcoholic fatty liver disease. World J Gastroenterol 2016;22:1008410092.

-9 Fernandes-Freitas I, Owen BM: Metabolic roles of endocrine fibroblast growth factors. Curr Opin Pharmacol 2015;25:30-35.

10 Nakayama Y, Masuda Y, Ohta H, Tanaka T, Washida M, Nabeshima Y, Miyake A, Itoh N, Konishi M: FGF21 regulates T-cell development in the neonatal and juvenile thymus. Sci Rep 2017;7:1-14.

-11 Dutchak PA, Katafuchi T, Bookout AL, Choi JH, Yu RT, Mangelsdorf DJ, Kliewer SA: Fibroblast growth factor-21 regulates PPAR $\gamma$ activity and the antidiabetic actions of thiazolidinediones. Cell 2012;148:556567.

12 Hondares E, Iglesias R, Giralt A, Gonzalez FJ, Giralt M, Mampel T, Villarroya F: Thermogenic activation induces FGF21 expression and release in brown adipose tissue. J Biol Chem 2011;286:12983-12990

13 Potthoff MJ, Kliewer SA, Mangelsdorf DJ: Endocrine fibroblast growth factors 15/19 and 21: from feast to famine. Genes Dev 2012;26:312-324.

14 Huang Z, Xu A, Cheung BM: The Potential Role of Fibroblast Growth Factor 21 in Lipid Metabolism and Hypertension. Curr Hypertens Rep 2017;19:2-6.

-15 Colak Y, Yesil A, Mutlu HH, Caklili OT, Ulasoglu C, Senates E, Takir M, Kostek O, Yilmaz Y, Yilmaz EF, Tasan G, Tuncer I A: Potential treatment of non-alcoholic fatty liver disease with SIRT1 activators. J Gastrointestin Liver Dis 2014;23:311-319.

16 Liu X, Gao Y, Li M, Geng C, Xu H, Yang Y: Sirt1 mediates the effect of the heme oxygenase inducer, cobalt protoporphyrin, on ameliorating liver metabolic damage caused by a high-fat diet. J Hepatol 2015:63:713721.

17 Ponugoti B, Kim DH, Xiao Z, Smith Z, Miao J, Zang M:SIRT1 deacetylates and inhibits SREBP-1C activity in regulation of hepatic lipid metabolism. J Biol Chem 2010;285:33959-33970.

- 18 Zhang Y, Geng C, Liu X, Li M, Gao M, Liu X, Fang F, Chang Y:Celastrol ameliorates liver metabolic damage caused by a high-fat diet through Sirt1. Mol Metab 2017;6:138-147.

19 Hardy T, Anstee QM, Day CP:Nonalcoholic fatty liver disease:new treatments. Curr Opin Gastroenterol 2015;31:175-183.

20 Sasidharan SR, Joseph AJ, Anandakumar S, Venkatesan V, Madhavan CNA, Agarwa A: Ameliorative potential of tamarindusindica on high fat dietinduced nonalcoholic fatty liver disease in rats. Sci World J 2014;1-10.

21 Murakami S: Role of taurine in the pathogenesis of obesity. Mol Nutr Food Res 2015; 59:1353-1363.

22 Sagara M, Murakami S, Mizushima S, Liu L: Taurine in 24-hour urine is inversely related to cardiovascular risks in middle aged subjects of 50 populations in the world. Adv Exp Med Biol 2015;2:623-636. 


\section{Cellular Physiology Cell Physiol Biochem 2017;43:644-659

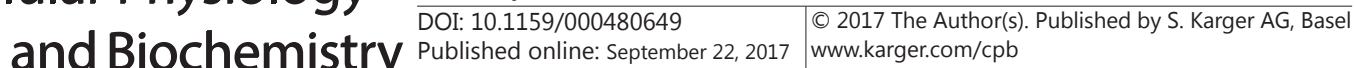

Abd Elwahab et al.: Role of SIRT1/ FGF-21 in Hepatoprotective Effect of Taurine in Experimental NAFLD

-23 Sampey BP, Vanhoose AM, Winfield HM, Freemerman AJ, Muehlbauer MJ, Fueger PT, Newgard CB, Makowski L: Cafeteria diet is a robust model of human metabolic syndrome with liver and adipose inflammation: comparison to high-fat diet. Obesity(Silver Spring)2011;19:1109-1117.

24 Soliman HM, Selim AO:Role of hepatic stellate cells in fibrogenesis in a model of pomegranate-treated fatty liver induced by junk food in male albino rats immunohistochemical and electron microscopic study. Egypt J Hist 2012;35:54-66.

25 Acharya M, Lau-Cam CA: Comparison of the protective actions of Nacetylcysteine, hypotaurine and taurine against acetaminophen-induced hepatotoxicity in the rat. J Biomed Sci 2010:17:S1-11.

26 Li Z, Henning SM, Zhang Y, Zerlin A, Li L, Gao K: Antioxidant-rich spice added to hamburger meat during cooking results in reduced meat, plasma and urine malondialdehyde concentrations. Am J Clin Nutr 2010;91:1180-1184.

27 Ochei J, Kolhatkar A: Medical Laboratory Science: Theory and Practice. Tata Mc Graw-Hill Publishing Company, New Delhi 2005, pp 281-283.

28 Sharma A, Artiss JD, Zak B: A method for the sequential colorimetric determination of serum triglycerides and cholesterol. Clin Biochem 1987;20:167-172.

29 Grillo F, Izzo C, Mazzotti G, Murador E: Improved method for determination of high-density-lipoprotein cholesterol II. Enzymic determination of cholesterol in high-density lipoprotein fractions with a sensitive reagent. Clin Chem 1981,27:375-379.

30 Friedwald WT, Levy RI, Fredrickson DS: Estimation of the concentration of low density lipoprotein cholesterol in plasma, without use of the preparative ultracentrifuge. Clin Chem 1975;18:499-502.

31 Tinder P: Determination of glucose in blood using glucose oxidase with alternative oxygen acceptor. Annals Clin Biochem1969;6:24-27.

-32 Starr JI, Mako ME, Juhn D, Rubenstein AH: Measurement of serum pro-insulin-like material: cross reactivity of porcine and human proinsulin. J Lab Clin Med 1978;91:691-692.

33 Matthews DR, Hosker JP, Rudenski AS, Naylor BA, Treacher DF, Turner RC: Homeostasis model assessment: insulin resistance and beta-cell function from fasting serum glucose and insulin concentrations in man. Diabetologia 1985;28:412-419.

34 Suominen P: Evaluation of an Enzyme Immunometric Assay to measure serum adiponectin concentration. Clin Chem 2004;50:219-221.

35 Ahmed MB, Ahmed MI, Meki AR, AbdRaboh N: Neurotoxic effect of lead on rats: Relationship to Apoptosis. Int J Health Sci 2013;7:192-199.

-36 Buege JA, Aust, SD: Microsomal lipid peroxidation. Methods Enzymol 1978;52:302-310.

37 Smith MR: Direct evidence for an intracellular role for tumor necrosis factor-alpha1. Microinjection of tumor necrosis factor kills target cells.J Immunol 1990;144:162-169.

38 Howard M, Harada N: in Nicola NA (ed): Guidebook to cytokines and their receptors. Oxford University Press, New York, 1994, pp 44.

-39 Talanian RV, Quinlan C, Trautz S, Hackett MC, Mankovich J, Banach D, Ghayur T: Substrate specificities of caspase family proteases, J Biol Chem 1997;272:9677-9682.

-40 Müller M, Beekman M, Steegenga WT: Fibroblast growth factor 21 reflects liver fat accumulation and dysregulation of signaling pathways in the liver of C57BL/6J mice. Sci Rep 2016;29,6:30484.

41 Deng X Q,Chen LL, Li N X: The expression of SIRT1 in nonalcoholic fatty liver disease induced by high-fat diet in rats. Liver Int 2007;27:708-715.

42 Mariani S, Fiore D, Basciani S, Persichetti A, Contini S, Lubrano C, Salvatori, L, Lenzi A, Gnessi L: Plasma levels of SIRT1 associate with non-alcoholic fatty liver disease in obese patients. Endocrine 2015;49:711716.

43 Banks AS, Kon N, Knight C, Matsumoto M, Gutiérrez-Juárez R, Rossetti L, Gu W, Accili1 D: SirT1 gain of function increases energy efficiency and prevents diabetes in mice.Cell Metab 20088:333-341.

44 Yan H, Xia M, Chang X, Xu Q, Bian H, Zeng M, Rao S, Yao X, Tu Y, Jia W, Gao X: Circulating fibroblast growth factor 21 levels are closely associated with hepatic fat content:a cross-sectional study. PloS ONE 2011;6:e24895.

45 Wree A, Schlattjan M, Bechmann LP: Adipocyte cell size, free fatty acids and apolipoproteins are associated with non-alcoholic liver injury progression in severely obese patients. Metab Clin Exp 2014;63:1542-1552.

-46 Yu HY, Xia FZ, Lam KSL: Circadian rhythm of circulating fibroblast growth factor 21 is related to diurnal changes in fatty acids in humans. Clin Chem 2011;57:691-700. 


\section{Cellular Physiology Cell Physiol Biochem 2017;43:644-659

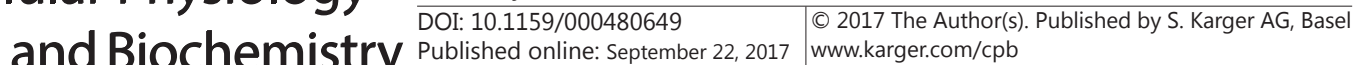 and Biochemistry Published online: September 22, 2017 www.kar}

Abd Elwahab et al.: Role of SIRT1/ FGF-21 in Hepatoprotective Effect of Taurine in Experimental NAFLD

-47 Qin Y, Zhou Y, Chen SH, Zhao XL, Ran L, Zeng XL, Wu Y, Chen JL, Kang C, Shu FR, Zhang QY, Mi MT: Fish Oil Supplements Lower Serum Lipids and Glucose in Correlation with a Reduction in Plasma Fibroblast Growth Factor 21 and Prostaglandin E2 in Nonalcoholic Fatty Liver Disease Associated with Hyperlipidemia: A Randomized Clinical Trial. PloS One 2015;30:10.

-48 Terashima Y, Nishiumi S, Minami A, Kawano Y, Hoshi N, Azuma T, Yoshida M: Metabolomics-based search for therapeutic agents for non-alcoholic steatohepatitis. Arch Biochem Biophys 2014;555-556.

-49 Li Y, Wong K, Giles A, Jiang J, Lee JW, Adams AC, Kharitonenkov A, Yang Q Gao B, Guarente L, Zang M: Hepatic SIRT1 attenuates hepatic steatosis and controls energy balance in mice by inducing fibroblast growth factor 21. Gastroenterology 2014;146:539-549.

50 Planavila A, Redondo-Angulo I, Ribas F, Garrabou G, Casademont J, Giralt M, Villarroya F. Fibroblast growth factor 21 protects the heart from oxidative stress. Cardiovasc Res 2015,106:19-31.

-51 Tarantino G, Finelli C, Scopacasa F, Pasanisi F, Contaldo F, Capone D, Savastano S. Circulating levels of sirtuin 4, a potential marker of oxidative metabolism, related to coronary artery disease in obese patients suffering from NAFLD, with normal or slightly increased liver enzymes. Oxid Med Cell Longev.2014;2014:920676,DOI: 10.1155/2014/920676

-52 Yang M, Xu D, Liu Y: Combined serum biomarkers in non-invasive diagnosis of non-alcoholic steatohepatitis. PLoS ONE 2015;10 (6):e0131664,DOI:10.1371.

-53 Tsuboyama-Kasaoka N, Shozawa C, Sano K, Kamei, Y: Taurine (2-aminoethanesulfonic acid) deficiency creates a vicious circle promoting obesity. Endocrinology 2006;147:3276-3284.

-54 Pina-Zentella G, de Rosa-Cuevas G, Va 'Zquez-Meza H, Pina E, de Pina MZ: Taurine in adipocytes prevents insulin-mediated H(2)o(2) generation and activates Pka and lipolysis. Amino Acids 2011;42:1927-1935.

-55 Chan DC, Watts GF, Gan S, Wong ATYE, Ooi MM, Barrett PHR: Nonalcoholic fatty liver disease as the transducer of hepatic oversecretion of very-low-density lipoprotein-apolipoprotein B-100 in Obesity. Arteriosclerosis Thromb Vasc Biol 2010;30:1043-1050.

-56 Xi Y, Wu M, Li H, Dong S, Luo E, Gu M, Shen X, Jiang Y, Liu Y, Liu H: Baicalin Attenuates High Fat Diet-Induced Obesity and Liver Dysfunction: Dose-Response and Potential Role of CaMKK $\beta /$ AMPK/ACC Pathway.Cell Physiol Biochem 2015,35:2349-2359.

-57 Engelmann G, Hoffmann GF, Grulich-Henn J, Teufel U: Alanine aminotransferase elevation in obese infants and children: a marker of early onset non alcoholic fatty liver disease. Hepatitis Monthly 2014;14(4):e14112,DOI:10.5812

-58 Girish C, Pradhan SC: Hepatoprotective activities of picroliv, curcumin, and ellagic acid compared to silymarin on carbon-tetrachloride-induced liver toxicity in mice. J Pharmacol Pharmacother 2012;3:149155.

-59 Murakami S, Sakurai T, Tomoike H, Sakono S, Nasu T, Fukuda N: Prevention of hypercholesterolemia and atherosclerosis in the hyperlipidemia- and atherosclerosis- prone Japanese (LAP) quail by taurine supplementation. Amino Acids 2010;38:271-278.

-60 Fukuda N, Ayako Y, Oshitama A, Satomi S, Ugita N, Michiko F: Dietary taurine reduces hepatic secretion of cholesteryl ester and enhances fatty acid oxidation in rats fed a high-cholesterol diet. J Nutr Sci Vitaminol 2011;57:144-149.

-61 Lam NV, Chen W, Suruga K, Nishimura N, Goda T, Yokogoshi H: Enhancing effect of taurine on CYP7A1 mRNA expression in Hep G2 cells. Amino Acids 2006;30:43-48.

62 Nishimura N, Yamamoto T, Ota T: Taurine feeding inhibits bile acid absorption from the ileum in rats fed a high cholesterol and high fat diet. Adv Exp Med Biol 2009;643:285-291.

63 Kotosai M, Shimada S, Kanda M, Terao J: Plasma HDL reduces nonesterified fatty acid hydroperoxides originating from oxidized LDL: a mechanism for its antioxidant ability. Lipids 2013;48:569-578.

64 Milnar B, Marc J, Janez A, Pfeifer M: Molecular mechanisms of insulin resistance and associated diseases. Clin Chim Acta 2007;375:20-35.

65 Stanhope KL, Havel PJ: Fructose consumption: potential mechanisms for its effects to increase visceral adiposity and induce dyslipidemia and insulin resistance. Curr Opin Lipidol 2008;19:16-24.

-66 El Mesallamy HO, El-Demerdash E, Hammad LN, El Magdoub, HM: Effect of taurine supplementation on hyperhomocysteinemia and markers of oxidative stress in high fructose diet induced insulin resistance. Diabetol Metab Synd 2010;2:1-11. 


\section{Cellular Physiology Cell Physiol Biochem 2017;43:644-659 \begin{tabular}{l|l|l|l|l} 
DOI: 10.1159/000480649 & 2017 The Author(s). Published by S. Karger AG, Basel \\
and Biochemistry
\end{tabular} and BiOChemistry Published online: September 22, 2017}

Abd Elwahab et al.: Role of SIRT1/ FGF-21 in Hepatoprotective Effect of Taurine in Experimental NAFLD

67 Khan R A, Kapur P, Jain A, Farah F, Bhandari1 U: Effect of orlistat on periostin, adiponectin, inflammatory markers and ultrasound grades of fatty liver in obese NAFLD patients. Ther Clin Risk Manag 2017;13:139149.

68 You JS, Zhao X, Kim SH, Chang KJ: Positive correlation between serum taurine and adiponectin levels in high-fat diet-induced obesity rats. Adv Exp Med Biol 2013;776:105-111.

-69 Rosa FT, Freitas E C, Deminice R, Jordao, AA, Marchini JS: Oxidative stress and inflammation in obesity after taurine supplementation:a double-blind, placebo-controlled study. Eur J Nutr 2014;53:823-830.

-70 Kim K S, Ji HI, Chung H, Kim C, Lee SH, Lee YA, Yang HI, Yoo MC, Hong SJ: Taurine chloramine modulates the expression of adipokines through inhibition of the STAT-3 signaling pathway in differentiated human adipocytes. Amino Acids 2013;45:1415-1422.

71 Mao Y, Cheng J, Yu F, Li H, Guo C, Fan X: Ghrelin Attenuated Lipotoxicity via Autophagy Induction and Nuclear Factor-кB Inhibition. Cell Physiol Biochem 2015,37:563-576.

72 Derosa G, Maffioli P, Sahebkar A: Improvement of plasma adiponectin, leptin and C-reactive protein concentrations by orlistat: a systematic review and meta-analysis. Br J Clin Pharmacol 2016;81:819-834.

73 Lak S, Ostadrahimi A, Nagili B, Asghari-Jafarabadi M, Beigzali, S, Salehi F, Djafarzadeh R: Anti-Inflammatory effect of taurine in burned patients. Adv Pharm Bull 2015;5:531-536.

74 Su Y, Fan, W, Ma Z, Wen X, Wang W, Wu Q, Huang H: Taurine improves functional and histological outcomes and reduces inflammation in traumatic brain injury. Neuroscience 2014;266:56-65.

75 Yang L, Tang J, Chen H, Ge D, Sui T, Que J, Cao X, Ge Y: Taurine Reduced Epidural Fibrosis in Rat Models after Laminectomy via Downregulating EGR1. Cell Physiol Biochem 2016,38:2261-2271.

76 Sayed AA: Ferulsinaic acid modulates SOD, GSH, and antioxidant enzymes in diabetic kidney. Evid Based Complement Alternat Med 2012;2012:580104:1-9

77 Li J, Wei X, Xie Q, Hoa Pham TT, Wei J, He P, Jiao Y, Xu X, Giang Nguyen TH, Wen Q Huang R: Protective Effects of 2-Dodecyl-6-Methoxycyclohexa-2,5 -Diene-1,4-Dione Isolated from Averrhoa Carambola L. (Oxalidaceae) Roots on High-Fat Diet-Induced Obesity and Insulin Resistance in Mice. Cell Physiol Biochem 2016,40:993-1004.

78 Nobili V, Donati B, Panera N, Vongsakulyanon A, Alisi A, Dallapiccola B, Valenti L: A 4-polymorphism risk score predicts steatohepatitis in children with nonalcoholic fatty liver disease. J Pediatr Gastroenterol Nutr 2014;58:632-636.

79 Das J, Ghosh J, Manna P, Sil P C: Acetaminophen induced acute liver failure via oxidative stress and JNK activation: protective role of taurine by the suppression of cytochrome P450 2E1. Free Radic Res 2010;44:340-355.

-80 Parvez S, Tabassum H, Banerjee BD, Raisuddin S: Taurine prevents tamoxifen-induced mitochondrial oxidative damage in mice. Basic Clin Pharmacol Toxicol 2008;102:382-387.

81 Jong CJ, Azuma J, Schaffer S: Mechanism underlying the antioxidant activity of taurine: prevention of mitochondrial oxidant production. Amino Acids 2012;42:2223-2232.

82 Youle RJ, Strasser A: The BCL-2 protein family:opposing activities that mediate cell death. Nat Rev Mol Cell Biol 2008;9:47-59.

83 Tarantino G, Scopacasa F, Colao A, Capone D, Tarantino M, Grimaldi E, Savastano S: Serum Bcl-2 concentrations in overweight-obese subjects with nonalcoholic fatty liver disease. World J Gastroenterol 2011,17(48):5280-8.

84 Wei Y,Wang D, Gentile CL, Pagliassotti MJ: Reduced endoplasmic reticulum luminal calcium links saturated fatty acid-mediated endoplasmic reticulum stress and cell death in liver cells. Mol Cell 2009;331:31-40.

85 Malhi H, Gores G J: Molecular mechanisms of lipotoxicity in nonalcoholic fatty liver disease. Semin Liver Dis 2008;28:360-369.

86 Guha P, Dey A, Sen R, Chatterjee M, Chattopadhyay S, Bandyopadhyay SK: Intracellular GSH depletion triggered mitochondrial Bax translocation to accomplish resveratrol-induced apoptosis in the U937 cell line. J Pharmacol Exp Ther 2011;336:206-214.

87 Watanabe M, Fuda H, Okabe H, Joko S, Miura Y, Hui SP, Min Y, Hamaoka N, Miki E, Chiba H: Oyster extracts attenuate pathological changes in non-alcoholic steatohepatitis (NASH) mouse model. J Functional Foods 2016;20:516-531.

-88 Gentile CL, Nivala AM, Gonzales JC, Pfaffenbach KT,Wang D,Wei Y,Jiang H,Orlicky DJ, Petersen DR, Pagliassotti MJ, Maclean KN: Experimental evidence for therapeutic potential of taurine in the treatment of nonalcoholic fatty liver disease. Am J Physiol 2011;30:1710-1722. 